\title{
Comparison of Six Statistics of Genetic Association Regarding Their Ability to Discriminate between Causal Variants and Genetically Linked Markers
}

\author{
Justo Lorenzo Bermejo ${ }^{\mathrm{a}, \mathrm{b}} \quad$ Alfonso Garcia Perez ${ }^{\mathrm{c}} \quad$ Andreas Brandt $^{\mathrm{b}}$ \\ Kari Hemminki ${ }^{\text {b, }}$ Abigail G. Matthews ${ }^{e, f}$

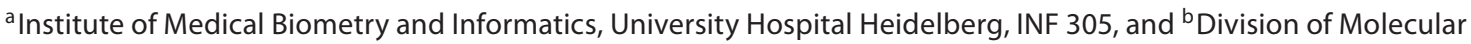 \\ Genetic Epidemiology, German Cancer Research Center, INF 520, Heidelberg, Germany; 'Department of Statistics, \\ Spanish Open University (UNED), Madrid, Spain; ${ }^{\mathrm{d} C e n t e r}$ for Primary Care Research, Lund University, Malmö, \\ Sweden; ${ }^{e}$ Ott Laboratory, Rockefeller University, New York, N.Y., and ${ }^{\mathrm{f}}$ The EMMES Corporation, Rockville, Md., USA
}

\section{Key Words}

Common-disease common-variant hypothesis - Markers • Causal susceptibility variants - Case-control study · Genetic association - Familial relative risk

\begin{abstract}
Objectives: Genome-wide association (GWA) studies still rely on the common-disease common-variant hypothesis since the assumption is associated with increased power. In GWA studies, polymorphisms are genotyped and their association with disease is investigated. Most of the identified associations are indirect and reflect a shared inheritance of the genotyped markers and genetically linked causal variants. We have compared six statistics of genetic association regarding their ability to discriminate between markers and causal susceptibility variants, including a probability value (Pval) and a Bayes Factor (BF) based on logistic regression, and the attributable familial relative risk (FRR). Methods: We carried out a simulation-based sensitivity analysis to explore several conceivable scenarios. Theoretical results were illustrated by established causal associations with age-related macular degeneration and by using imputed data based on HapMap for a case-control study of breast cancer. Results:
\end{abstract}

Our data indicate that a representation of genetic association by FRRs and BFs generally facilitates the distinction of causal variants. The FRR showed the best discriminative power under most investigated scenarios, but no single statistic outperformed the others in all situations. For example, rare moderate- to low-penetrance variants (allele frequency: $1 \%$, dominant odds ratio: $\leq 2.0)$ seem to be best discriminated by BFs. Conclusions: Present results may help to fully utilize the data generated in association studies that take advantage of next generation sequencing and/or multiple imputation based on the 1000 Genomes Project.

Copyright $\odot 2011$ S. Karger AG, Basel

\section{Introduction}

Genome-wide association (GWA) studies often focus on variants with an allele frequency over $1-5 \%$ relying on the common-disease common-variant hypothesis [1]. Hundreds of thousands of single nucleotide polymorphisms (SNPs) are genotyped and then the association between these markers and disease status is tested. Most of the identified associations are assumed to be the result of a co-inheritance of the detected marker and the causal

\section{KARGER}

Fax +41613061234 E-Mail karger@karger.ch www.karger.com
(C) 2011 S. Karger AG, Base

$0001-5652 / 11 / 0722-0142 \$ 38.00 / 0$

Accessible online at:

www.karger.com/hhe
Justo Lorenzo Bermejo

Institute of Medical Biometry and Informatics

University Hospital Heidelberg

INF 305, DE-69120 Heidelberg (Germany)

Tel. +496221 564 180, E-Mail Justo.Lorenzo@ imbi.uni-heidelberg.de 
variant(s) (see fig. 1). The ultimate goal of GWA studies is to yield information regarding disease aetiology, treatment and possible prevention. For example, it may well be that the detected marker associates with a causal variant that is involved in angiogenesis, and it may be that the identification of that association translates into the development of a new treatment.

The first aim of GWA studies is to detect association signals, commonly relying on 'significance testing' and summarizing results by probability values (Pvals). When more concrete hypotheses and more refined studies have been set up (for example, focusing on a specific chromosomal region and incorporating genotypes from public data repositories), the subsequent goal is to quantify and model associations, and to identify causal variants underlying the signals from GWA studies (fig. 1). The investigated genotypes are usually ranked according to plausibility of causal association, and most often rankings are based on frequentist Pvals, although Bayes Factors (BFs) are increasingly common [2]. An alternative would be to move from a 'significance testing' paradigm to an 'effect size' framework, with the advantage of easier interpretability of the statistics [3-5]. For example, it is in principle easier to interpret that ' $5 \%$ of the cases can be attributed to the investigated variant' than 'the probability of the variant being identified as associated with disease in the absence of association is $0.1 \%$. The present study investigates four statistics in addition to the most common Pval and BF: the population-attributable fraction (PAF; relative change of disease incidence if a susceptibility variant was not present), the familial relative risk (FRR; contribution of a variant to disease aggregation among siblings), the pseudo $\mathrm{R}^{2}$ (predictive ability of the genetic variant) and the area under the receiver operating characteristics curve (AUC; power of the variant to discriminate between cases and controls).

Our strategy is simple: we calculate the expected values of the six statistics for markers and causal variants, and use the relative change in the statistics as a measure of discriminative power.

\section{Methods}

Investigated Statistics of Genetic Association

We consider six measures of genetic association: PAF, FRR, BF, Pval, AUC and pseudo $\mathrm{R}^{2}$.

Population Attributable Fraction

The PAF reflects the reduction in disease incidence that would be observed if the population was not exposed to a given risk factor. In the present context, the PAF measures the relative reduc-

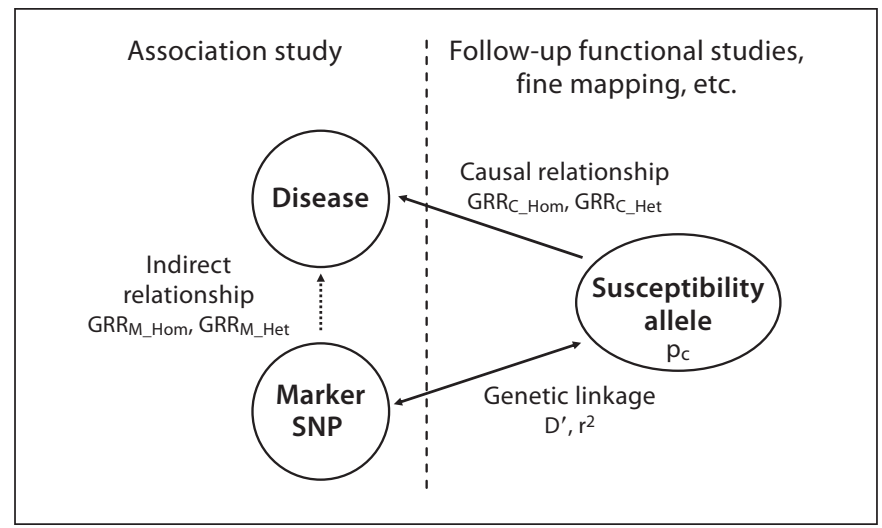

Fig. 1. Rationale for the identification of disease susceptibility variants relying on the common-disease common-variant hypothesis.

tion of disease incidence when a high-risk allele is not present in a population. Let the frequency of the high-risk allele (M) be denoted by $p$, the relative risk of disease for high-risk allele homozygotes (MM) compared to low-risk allele homozygotes $(\mathrm{mm})$ by $\mathrm{GRR}_{\text {Hom, }}$, the relative risk for heterozygotes $(\mathrm{Mm})$ compared to low-risk allele homozygotes by $\mathrm{GRR}_{\mathrm{Het}}$ and the disease prevalence among low-risk allele homozygotes by $\kappa_{0}$. We premise that different genotypes have equal fitness even if the variant has a strong effect on the risk of disease and assume Hardy-Weinberg equilibrium (HWE). Then, the probability that an individual in the population is wild-type homozygotic $(\mathrm{G}=\mathrm{mm})$ and affected $(\mathrm{A}=1)$ is:

$$
\operatorname{Pr}(\mathrm{G}=\mathrm{mm}, \mathrm{A}=1)=(1-\mathrm{p})^{2} \kappa_{0} .
$$

Similarly,

$$
\begin{aligned}
& \operatorname{Pr}(\mathrm{G}=\mathrm{Mm}, \mathrm{A}=1)=2 \mathrm{p}(1-\mathrm{p}) \mathrm{GRR}_{\mathrm{Het}} \kappa_{0} \text { and } \\
& \operatorname{Pr}(\mathrm{G}=\mathrm{MM}, \mathrm{A}=1)=\mathrm{p}^{2} \mathrm{GRR}_{\mathrm{Hom}} \kappa_{0} .
\end{aligned}
$$

The prevalence of the disease in the population is then:

$$
\begin{aligned}
\kappa= & \Sigma_{\mathrm{G}} \operatorname{Pr}(\mathrm{A}=1, \mathrm{G})=\kappa_{0}\left[(1-\mathrm{p})^{2}+2 \mathrm{p}(1-\mathrm{p}) \mathrm{GRR}_{\text {Het }}\right. \\
& \left.+\mathrm{p}^{2} \mathrm{GRR}_{\text {Hom }}\right] .
\end{aligned}
$$

Therefore the PAF is given by:

$$
\operatorname{PAF}=\left(\kappa-\kappa_{0}\right) / \kappa .
$$

Familial Relative Risk

The FRR is the ratio of the disease probability for a relative of a patient and the overall disease prevalence. FRRs can be inferred for different types of relationship. We consider here the extent to which a particular genetic locus contributes to disease aggregation within sib-pairs, which is a statistic of genetic association that is also referred to as the attributable sibling relative risk. FRR calculation was first described in an early paper by James, and more recently by Risch [6, 7]. The 'excess FRR' (1 - FRR) is given by:

$$
1-\mathrm{FRR}=\left(1 / 2 \mathrm{~V}_{\mathrm{a}}+1 / 4 \mathrm{~V}_{\mathrm{d}}\right) / \mathrm{K}^{2},
$$


where $\mathrm{V}_{\mathrm{a}}$ is the additive genetic variance divided by $\kappa_{0}^{2}, \mathrm{~V}_{\mathrm{d}}$ is the dominance genetic variance divided by $\kappa_{0}^{2}$ and $\mathrm{K}$ is the population prevalence divided by $\kappa_{0}$. It can be shown that:

$$
\begin{aligned}
& \mathrm{V}_{\mathrm{a}}=2 \mathrm{p}(1-\mathrm{p})\left[(1-\mathrm{p})\left(1-\mathrm{GRR}_{\mathrm{Het}}\right)-\mathrm{p}\left(\mathrm{GRR}_{\mathrm{Hom}}-\mathrm{GRR}_{\mathrm{Het}}\right)\right]^{2}, \\
& \mathrm{~V}_{\mathrm{d}}=\mathrm{p}^{2}(1-\mathrm{p})^{2}\left[1+\mathrm{GRR}_{\mathrm{Hom}}-2 \mathrm{GRR}_{\mathrm{Het}}\right]^{2} \text { and } \\
& \mathrm{K}=\mathrm{p}^{2} \mathrm{GRR}_{\mathrm{Hom}}+2 \mathrm{p}(1-\mathrm{p}) \mathrm{GRR}_{\mathrm{Het}}+(1-\mathrm{p})^{2} .
\end{aligned}
$$

Thus, an advantageous property of the FRR is that it only depends on $\mathrm{p}, \mathrm{GRR}_{\mathrm{Hom}}$ and $\mathrm{GRR}_{\mathrm{Het}}$, that is, the excess FRR is independent of the disease prevalence among low-risk homozygotes $\left(\kappa_{0}\right)[8-10]$.

\section{Bayes Factor}

The BF quantifies evidence of genetic association from a Bayesian viewpoint. The BF can be defined as the ratio of the probability of observed data under association to its probability under the null hypothesis (absence of association). With this definition, a large $\mathrm{BF}$ value implies evidence in favour of association. We use here a base 10 logarithmic transformation of the $\mathrm{BF}\left(\log _{10} \mathrm{BF}\right)$. We consider a logistic regression model where the affection status $\mathrm{A}$ depends on the individual genotype (three-genotype association model). Under this model, $\mathrm{A}_{i} \sim \operatorname{Bernoulli}\left(\pi_{\mathrm{i}}\right)$ with

$$
\pi_{\mathrm{i}}=\exp \left(\mathbf{x}_{i}^{\prime} \boldsymbol{\beta}\right) /\left[1+\exp \left(\mathbf{x}_{i}^{\prime} \boldsymbol{\beta}\right)\right],
$$

where $\mathrm{x}_{i}$ is a column vector that contains the observed genotypes (mm, Mm, MM).

\section{Probability Value}

The global Pval for the genetic effect is the frequentist counterpart of the $\mathrm{BF}$ and is also based on the logistic regression model. A small Pval, and thus a large value of $-\log _{10} \mathrm{Pval}$, indicates statistical significance. It should be kept in mind that large $-\log _{10}$ Pvals do not automatically reflect large genetic effects, and that rare variants with large genetic effects may result in relatively low $-\log _{10}$ Pvals. BFs have been proposed to circumvent the limitations of Pvals, but the properties of other 'frequentist' alternatives, like measures of predictive performance of the model, have been less investigated.

Area under the Receiver Operating Characteristic Curve

The AUC evaluates the performance of the genotype as a predictor of affection status. In the present context of association studies, the AUC is calculated based on the true- and on the falsepositive rates for two values of the classification threshold 'number of high-risk alleles' ( $<1$ and $<2$ ). The higher the AUC, the better the performance, with 0.5 indicating random performance and 1.0 denoting perfect performance.

\section{Pseudo $\mathrm{R}^{2}$}

Several pseudo $\mathrm{R}^{2}$ statistics have been developed to evaluate the goodness-of-fit of logistic models, with higher values indicating better model fit. We explore here the Nagelkerke's pseudo $\mathrm{R}^{2}$ index (pseudo $\mathrm{R}^{2}$ ) as a measure of goodness-of-fit (pseudo $\mathrm{R}^{2}=1$ if the logistic regression model with genotypes perfectly predicts disease status) [11-14].

\section{Genetic Parameters for Causal Variants and Linked Markers}

In order to evaluate the ability of the six investigated statistics to discriminate between markers and causal susceptibility variants, we build on the common-disease common-variant hypothesis and consider that the risk of disease is conditionally independent of the marker genotype, given the genotype at the causal locus (fig. 1). We assume that an allele with frequency $\mathrm{p}_{\mathrm{C}}$ causes the disease according to known relative risks for heterozygotic $\left(\mathrm{GRR}_{\mathrm{C} \_\mathrm{Het}}\right)$ and homozygotic $\left(\mathrm{GRR}_{\mathrm{C} \_ \text {Hom }}\right)$ allele carriers. The two parameters $\mathrm{D}^{\prime}$ and $\mathrm{r}^{2}$ quantify the genetic association between causal allele $\mathrm{C}$ and marker M. Lewontin's D' assesses the extent of linkage disequilibrium (LD) relative to its maximum value at the given allele frequencies and the maximum value of $r^{2}$ also depends on the allele frequencies. We derive first the genetic parameters for the marker $\left(\mathrm{p}_{\mathrm{M}}, \mathrm{GRR}_{\mathrm{M}_{-} \text {Hom }}\right.$ and $G_{R R} R_{M_{-} H_{e t}}$ ) based on the genetic parameters for the causal allele and the linkage parameters. Calculation details are presented in the Appendix. In a second step, we examine the expected values of PAF, AUC, $\log _{10} \mathrm{BF}$, pseudo $\mathrm{R}^{2}$, $-\log _{10}$ Pval and FRR in a hypothetical case-control study. The relative change in the statistic of genetic association with decreasing LD is then used as the measure of discriminative power between marker and causal variant.

The expected excess FRR for the marker can be obtained by plugging the parameters $\mathrm{p}_{\mathrm{M}}, \mathrm{GRR}_{\mathrm{M}_{-} \text {Hom }}$ and $\mathrm{GRR}_{\mathrm{M}_{-} \text {Het }}$ into equation 2 . An additional parameter ( $\kappa_{0}$ or alternatively $\kappa$ ) needs to be considered to calculate the PAF according to equation 1 . In order to investigate the expected values of $\log _{10} \mathrm{BF}$, pseudo $\mathrm{R}^{2}$, $-\log _{10}$ Pval and AUC, the expected distributions of genotypes in a hypothetical case-control study that investigated the relationship between marker and disease are calculated under domi-

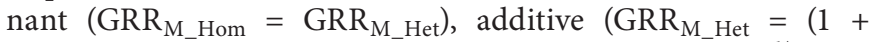
$\left.\left.\mathrm{GRR}_{\mathrm{M}_{-} \text {Hom }}\right) / 2\right)$, multiplicative $\left(\mathrm{GRR}_{\mathrm{M} \_ \text {Hom }}=\mathrm{GRR}_{\mathrm{M}_{-} \mathrm{Het}^{1 / 2}}\right)$ and recessive $\left(\mathrm{GRR}_{\mathrm{M} \_\mathrm{Het}}=1\right)$ penetrances. Assuming $\mathrm{HWE}$ in the controls $(\mathrm{A}=0)$, the expected distribution of genotypes is:

$$
\begin{aligned}
& \operatorname{Pr}(\mathrm{G}=\mathrm{mm} \mid \mathrm{A}=0)=\left(1-\mathrm{p}_{\mathrm{M}}\right)^{2}, \\
& \operatorname{Pr}(\mathrm{G}=\mathrm{Mm} \mid \mathrm{A}=0)=2 \mathrm{p}_{\mathrm{M}}\left(1-\mathrm{p}_{\mathrm{M}}\right) \text { and } \\
& \operatorname{Pr}(\mathrm{G}=\mathrm{MM} \mid \mathrm{A}=0)=\mathrm{p}_{\mathrm{M}}{ }^{2} .
\end{aligned}
$$

The expected distribution of genotypes in cases $(\mathrm{A}=1)$ satisfies:

$$
\mathrm{GRR}_{\mathrm{M} \_ \text {Hom }}=\operatorname{Pr}(\mathrm{A}=1 \mid \mathrm{G}=\mathrm{MM}) / \operatorname{Pr}(\mathrm{A}=1 \mid \mathrm{G}=\mathrm{mm})
$$

and

$$
\mathrm{GRR}_{\mathrm{M} \_ \text {Het }}=\operatorname{Pr}(\mathrm{A}=1 \mid \mathrm{G}=\mathrm{Mm}) / \operatorname{Pr}(\mathrm{A}=1 \mid \mathrm{G}=\mathrm{mm}) .
$$

\section{Simulations and Sensitivity Analysis}

Since the causal variants are expected to be rare, we assume a sample size of 5,000 cases and 5,000 controls in the reference scenario and investigate the expected distributions of genotypes by frequentist and Bayesian logistic regression. Pseudo $\mathrm{R}^{2},-\log _{10} \mathrm{Pval}$ and AUC were determined by the function $\mathrm{lrm}$ (R package Design). The function MCMClogit (R package MCMCpack) was used to generate 10,000 samples from the posterior distribution using a random walk Metropolis algorithm and the BayesFactor function in the same package was used to compute log marginal likelihoods for a model 'with' genotypic information compared to a model 'without' it, using a Laplace approximation. The reference scenario presumed a standard normal prior on the mean and $\mathrm{N}(0$, $1,000)$ priors on the two genetic effects. In this context, the mean represents the baseline log-odds of disease. A standard normal prior has been previously used for the mean and BFs seem to be relatively insensitive to this choice [15]. Regarding the genetic effects, our prior belief is centred on their absence. Note that, since $1.96 \times 1,000^{1 / 2} \approx 62$, the prior puts $95 \%$ probability on the broad interval for the log-odds of disease $[-62,62]$. The prior precision is the reciprocal of the prior variance (0.001) and represents the 
weight of the prior in the a posteriori distribution. These kinds of diffuse priors that have minimal impact on the final estimates are referred to as 'flat' priors in Bayesian statistics. The dependence of results on alternative parameter values (frequency, genotype relative risks and penetrance model of the causal variant, genetic linkage, sample size and prior precision) was investigated in a sensitivity analysis. Further, since genotype calling errors can introduce severe bias in GWA studies, their potential effect was assessed under two different models [16]. In the first error model, each genotype was randomly assigned to $5 \%$ of cases and controls using equal weights for $\mathrm{MM}, \mathrm{Mm}$ and $\mathrm{mm}$. In the second error model, a random genotype was assigned to $5 \%$ of heterozygote individuals, again sampling uniformly from the three genotypes.

\section{Illustration of Theoretical Results Based on Real Datasets}

In order to exemplify the practical relevance of theoretical results, the investigated statistics of genetic association were applied to two real datasets. The first dataset consisted of the supplementary material provided by Edwards et al. [17] (table S4) in their article 'Complement factor $\mathrm{H}$ polymorphism and age-related macular degeneration'. In that study, a significant association $\left(\mathrm{Pval}=4.95 \times 10^{-10}\right.$, per allele $\left.\mathrm{OR}=2.7\right)$ was found within the regulation of complement activation locus (polymorphism rs1061170, the frequency of the high-risk allele $C$ was 0.36 ). $R$ code to calculate the values of the six statistics for the dataset from Edwards et al. is available from the corresponding author.

The second dataset we considered has been previously used to assess the relationship between polymorphisms in the UCB9 gene of the SUMO-conjugating system and breast cancer risk [18]. Three variants (rs7187167, rs8052688 and rs11248866) were directly genotyped in 997 breast cancer patients and 994 matched controls. Approximately 110 variants in a $\pm 150-\mathrm{kb}$ region around genotyped SNPs were imputed based on HapMap data (Rel 24/ phase II Nov 08) relying on inference of haplotypes by means of the expectation-maximization (EM) algorithm [19]. In brief, missing alleles were excluded from the calculation of allele frequencies. In the E-step, the frequencies of partially missing genotypes were updated looping through all possible genotypes. In the M-step, all existing haplotypes that have alleles identical to the non-missing alleles of this haplotype were updated. Uncertainty in the imputed genotypes was taken into account by bootstrapping from the multinomial distribution of the expected genotypes given the observed, directly typed variants (1,000 replicates). Dünnebier et al. [21] summarized the relationship between imputed genotypes and risk of breast cancer by Pvals but we additionally consider here the other five statistics discussed above.

\section{Results}

\section{Simulations and Sensitivity Analysis}

Figure 2 shows the expected parameter values for a marker $\mathrm{M}$ genetically linked $\left(\mathrm{D}^{\prime}=1.00,0.95\right.$ or 0.90$)$ to a causal variant $\mathrm{C}\left(\mathrm{p}_{\mathrm{C}}=0.01\right)$. The relative risks and penetrance models for the causal variant are indicated in the left part of the figure. The value of $r^{2}$ is shown on the horizontal axis. Black lines represent the GRRs for the marker (scale on the left axes), which decrease with de- creasing $r^{2}$. Grey lines indicate the expected marker frequency ( $\mathrm{p}_{\mathrm{M}}$, scale on the right axes), which increases with decreasing $r^{2}$. We observe that a dominant penetrance model for the causal variant translates into a non-dominant penetrance model for the marker. Note that $\mathrm{D}^{\prime}$ constrains the domain of $\mathrm{r}^{2}$.

Table 1 shows the expected values for the six investigated statistics under the reference scenario $\left(\mathrm{p}_{\mathrm{C}}=0.01\right.$, $\mathrm{GRR}_{\mathrm{C}_{-} \mathrm{Hom}}=\mathrm{GRR}_{\mathrm{C} \_ \text {Het }}=5, \mathrm{D}^{\prime}=1$, sample size of 5,000 cases and 5,000 controls, prior precision $=0.001$ for the two genetic effects and $\kappa=0.1$ ). We considered $\mathrm{r}^{2}$ values from 0.8 to 1.0 (denoting causal variant) reflecting 'strong' LD (see for example, Hivert et al. [20]). The AUC does not depend on $r^{2}$ as demonstrated by the fact that it attains practically identical values for the marker and the causal variant. When $\mathrm{D}^{\prime}=1$, removal of the marker implies deletion of the causal variant, thus resulting in identical PAFs for different values of $r^{2}$. The $\log _{10} B F$ equals 55.7 for the causal variant $\left(r^{2}=1.0\right)$ and it decreases to 49.8 for $r^{2}=0.8$. The relative change in the statistic of genetic association is more relevant than its absolute value in order to discriminate between markers and causal variants. Under the first scenario, the relative reduction $\left(\mathrm{r}^{2}=0.8\right.$ versus $r^{2}=1.0$ ) varies from $0 \%$ (PAF and AUC) to $20 \%$ (excess FRR). These results are presented with more detail in figure 3a. The investigated statistics suggest a linear dependence on $\mathrm{r}^{2}$. The PAF and the AUC do not distinguish between marker and causal variant. The excess FRR discriminates the best of all six statistics. The relative decreases of pseudo $R^{2}$ and $\log _{10} B F$ are quite similar under the reference scenario, and they decrease at a faster rate than $-\log _{10}$ Pval as $r^{2}$ decreases.

In the sensitivity analysis, the assumed parameter values were varied one by one in order to investigate their impact on the discriminative power of the six statistics. Table 1 illustrates that additive causal variants are best discriminated by the excess FRR, followed by the $\log _{10} \mathrm{BF}$, pseudo $\mathrm{R}^{2}$ and $-\log _{10} \mathrm{Pval}$. The ranking of statistics according to discriminative power was markedly different for lower penetrances $\left(G R_{C_{-} H o m}=G R R_{C_{-} H e t}=2\right)$ as seen in the third scenario of table 1 . Detailed results are presented in figure $3 \mathrm{~b}$. Under this scenario, the $\log _{10} \mathrm{BF}$ discriminates best over the whole domain of $\mathrm{r}^{2}$, followed by excess FRR, the pseudo $\mathrm{R}^{2}$, $-\log _{10} \mathrm{Pval}$ and PAF/AUC $\left(r^{2}=0.8\right)$. Note that, due to the discrete nature of the expected number of cases and controls, the relationships between $\mathrm{r}^{2}$ and $-\log _{10} \mathrm{Pval}$, pseudo $\mathrm{R}^{2}$ and $\log _{10} \mathrm{BF}$ are neither smooth nor monotone. For example, figure $3 \mathrm{~b}$ shows a decrease of $-\log _{10}$ Pval from 7.14 to 6.95 when $r^{2}$ decreases from 0.92 to 0.91 , followed by an increase of $-\log _{10} \mathrm{Pval}$ 


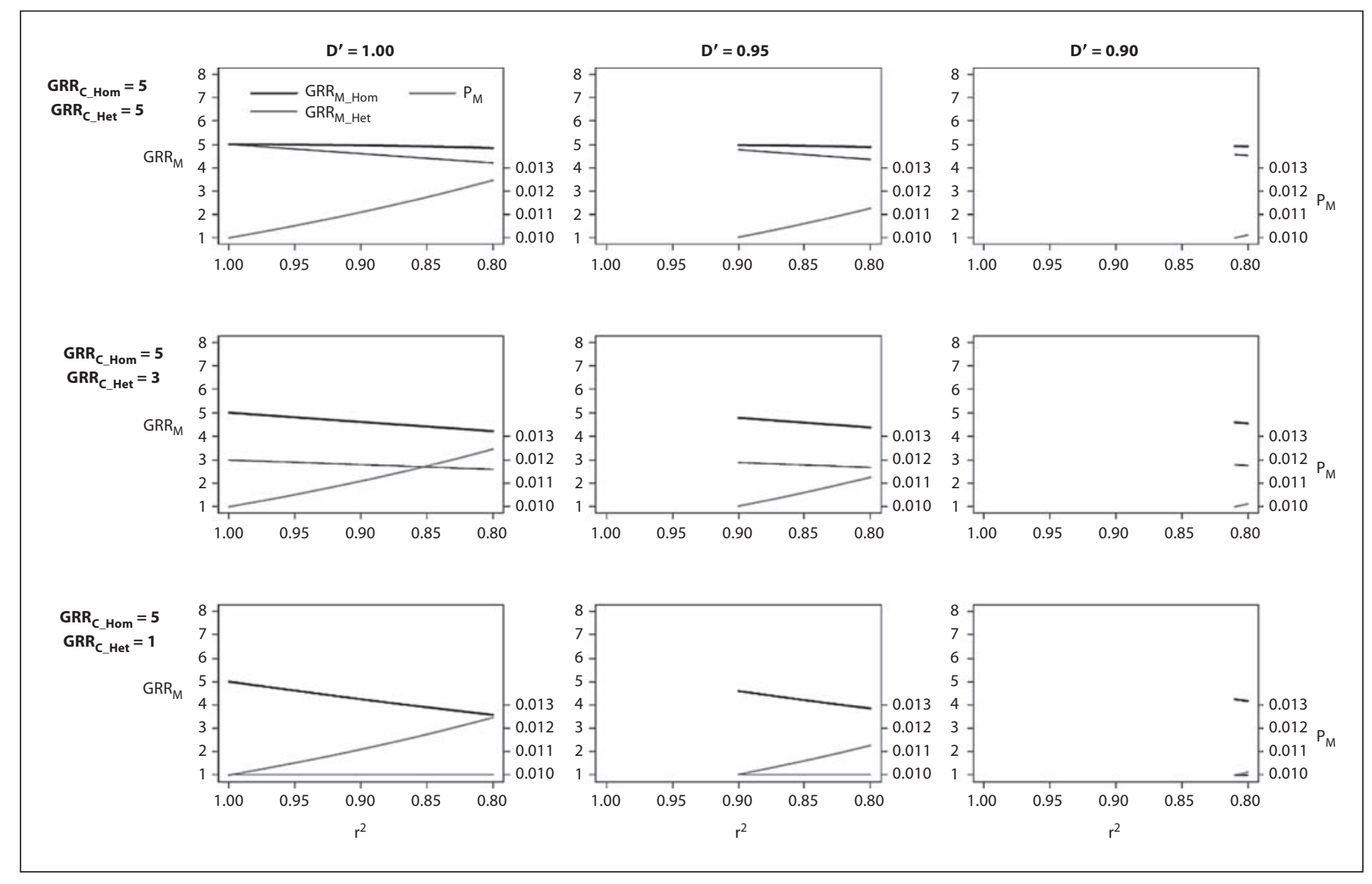

Fig. 2. Genotype relative risks and frequencies for a marker $M$ linked to a causal variant $C$ under several scenarios. Assumed parameters are shown in bold type.

Fig. 3. Comparison of the investigated statistics for a marker $\mathrm{M}$ genetically linked to a causal variant $C$. The assumed parameters were $\mathrm{p}_{\mathrm{C}}=0.01 ; \mathrm{D}^{\prime}=1 ; 5,000$ cases and 5,000 controls; prior precision $=0.001 ; \kappa=$ 0.1 and $\mathrm{GRR}_{\mathrm{C}_{\text {_Hom }}}=\mathrm{GRR}_{\mathrm{C}_{\text {HHet }}}=5$ (a) or alternatively GRR $\mathrm{C}_{\text {_Hom }}=\mathrm{GRR}_{\mathrm{C}_{-} \text {Het }}=2(\mathbf{b})$.

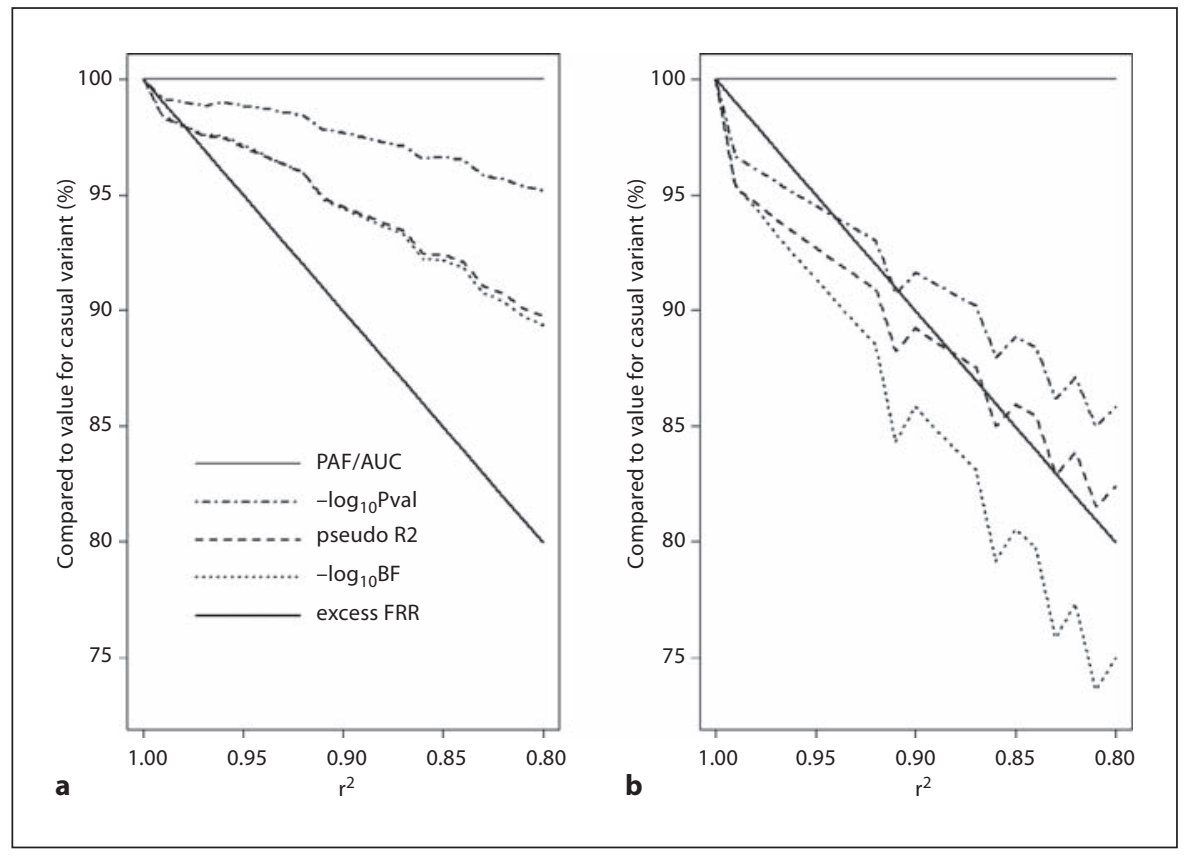

Lorenzo Bermejo/Garcia Perez/Brandt/ Hemminki/Matthews 
Table 1. Comparison of the investigated statistics of association for a marker genetically linked to a causal variant $\mathrm{C}$ under several genotype relative risks and genotyping error models

\begin{tabular}{|c|c|c|c|c|c|c|}
\hline \multirow{2}{*}{$\begin{array}{l}\text { Statistic of genetic } \\
\text { association }\end{array}$} & \multicolumn{2}{|c|}{$\mathrm{r}^{2}=1.0$} & \multicolumn{2}{|c|}{$\mathrm{r}^{2}=0.9$} & \multicolumn{2}{|c|}{$\mathrm{r}^{2}=0.8$} \\
\hline & value & $\begin{array}{l}\% \text { compared to } \\
\text { causal variant }\end{array}$ & value & $\begin{array}{l}\% \text { compared to } \\
\text { causal variant }\end{array}$ & value & $\begin{array}{l}\% \text { compared to } \\
\text { causal variant }\end{array}$ \\
\hline \multicolumn{7}{|c|}{$\mathrm{GRR}_{\mathrm{C} \_ \text {Hom }}=\mathrm{GRR}_{\mathrm{C} \_ \text {Het }}=5$, no genotyping error (reference scenario) } \\
\hline PAF & 7.37 & 100 & 7.37 & 100 & 7.37 & 100 \\
\hline AUC & 0.536 & 100 & 0.536 & 100 & 0.536 & 100 \\
\hline $\log _{10} \mathrm{BF}$ & 55.7 & 100 & 52.6 & 94 & 49.8 & 89 \\
\hline pseudo $\mathrm{R}^{2}$ & 0.035 & 100 & 0.033 & 95 & 0.032 & 90 \\
\hline$-\log _{10}$ Pval & 45.9 & 100 & 44.8 & 98 & 43.7 & 95 \\
\hline FRR & 1.13 & 100 & 1.12 & 90 & 1.11 & 80 \\
\hline \multicolumn{7}{|c|}{$\mathrm{GRR}_{\mathrm{C} \_H o m_{-}}=5, \mathrm{GRR}_{\mathrm{C} \_H e t_{\mathrm{H}}}=3$, no genotyping error } \\
\hline PAF & 3.85 & 100 & 3.85 & 100 & 3.85 & 100 \\
\hline AUC & 0.519 & 100 & 0.519 & 100 & 0.519 & 100 \\
\hline $\log _{10} \mathrm{BF}$ & 17.3 & 100 & 15.9 & 92 & 14.7 & 85 \\
\hline pseudo $\mathrm{R}^{2}$ & 0.013 & 100 & 0.012 & 92 & 0.012 & 86 \\
\hline$-\log _{10}$ Pval & 20.2 & 100 & 19.1 & 95 & 18.1 & 90 \\
\hline FRR & 1.04 & 100 & 1.03 & 90 & 1.03 & 80 \\
\hline \multicolumn{7}{|c|}{$\mathrm{GRR}_{\mathrm{C} \_ \text {Hom }_{\text {Hot }}}=\mathrm{GRR}_{\mathrm{C}_{-} \text {Het }}=2$, no genotyping error } \\
\hline PAF & 1.95 & 100 & 1.95 & 100 & 1.95 & 100 \\
\hline AUC & 0.510 & 100 & 0.510 & 100 & 0.510 & 100 \\
\hline $\log _{10} \mathrm{BF}$ & 4.7 & 100 & 4.1 & 86 & 3.6 & 75 \\
\hline pseudo $\mathrm{R}^{2}$ & 0.004 & 100 & 0.004 & 89 & 0.004 & 82 \\
\hline$-\log _{10}$ Pval & 7.7 & 100 & 7.0 & 92 & 6.6 & 86 \\
\hline FRR & 1.01 & 100 & 1.01 & 90 & 1.01 & 80 \\
\hline \multicolumn{7}{|c|}{$\mathrm{GRR}_{\mathrm{C}_{-} \text {Hom }}=\mathrm{GRR}_{\mathrm{C}_{-} \text {Het }}=5$, genotyping error rate $=0.05$ for all genotypes } \\
\hline PAF & 13.33 & 100 & 13.08 & 98 & 12.76 & 96 \\
\hline AUC & 0.534 & 100 & 0.534 & 100 & 0.534 & 100 \\
\hline $\log _{10} \mathrm{BF}$ & 24.3 & 100 & 23.6 & 97 & 22.9 & 94 \\
\hline pseudo $\mathrm{R}^{2}$ & 0.021 & 100 & 0.021 & 97 & 0.020 & 94 \\
\hline$-\log _{10}$ Pval & 26.7 & 100 & 26.2 & 98 & 25.6 & 96 \\
\hline FRR & 1.09 & 100 & 1.08 & 94 & 1.08 & 87 \\
\hline \multicolumn{7}{|c|}{$\mathrm{GRR}_{\mathrm{C}_{-} \text {Hom }}=\mathrm{GRR}_{\mathrm{C}_{-} \text {Het }}=5$, genotyping error rate $=0.05$ for heterozygotes } \\
\hline PAF & 7.37 & 100 & 7.33 & 99 & 7.36 & 100 \\
\hline AUC & 0.535 & 100 & 0.535 & 100 & 0.535 & 100 \\
\hline $\log _{10} \mathrm{BF}$ & 54.1 & 100 & 51.8 & 96 & 48.4 & 89 \\
\hline pseudo $\mathrm{R}^{2}$ & 0.034 & 100 & 0.033 & 96 & 0.031 & 90 \\
\hline$-\log _{10}$ Pval & 44.7 & 100 & 44.0 & 99 & 42.5 & 95 \\
\hline FRR & 1.13 & 100 & 1.12 & 90 & 1.11 & 79 \\
\hline
\end{tabular}

$\mathrm{p}_{\mathrm{C}}=0.01 ; \mathrm{D}^{\prime}=1 ; 5,000$ cases and 5,000 controls; prior precision $=0.001 ; \kappa=0.10$.

to 7.00 when $\mathrm{r}^{2}$ equals 0.90 . This discreteness does not affect the relationship between FRR and $\mathrm{r}^{2}$, which is smooth because the FRR does not directly depend on the sample size. The same holds for the PAF, and the AUC does not practically depend on $\mathrm{r}^{2}$.

Discrimination between Causal Variants and Linked Markers by Six Statistics
Figure 3 illustrates a key result from the present study. All investigated statistics show a nearly linear relationship with $\mathrm{r}^{2}$. However, the specific dependence of the statistic on $r^{2}$, measured by the relative change of the statistic or the slope of the depicted lines, varies according to

Hum Hered 2011;72:142-152 
multiple parameters (for example, the GRR) which are unknown in real applications, where several variants are simultaneously investigated. The larger the correlation between the statistic and $\mathrm{r}^{2}$, the easier it is to discriminate between markers and causal variants. We aim to identify those statistics showing the strongest dependence on $r^{2}$ in plausible real scenarios.

Results under multiplicative and recessive penetrance, alternative allele frequencies and additional data from the sensitivity analysis are provided as supplementary material (online supplementary table 1, www. karger.com/doi/10.1159/000332006). The $\log _{10} \mathrm{BF}$ and the FRR outperform the $-\log _{10} \mathrm{Pval}$ under a multiplicative inheritance model. Separate sample size calculations indicate that a recessive penetrance results in low statistical power $(0.21$, for type I error rate $=0.05)$, even assuming a large study (5,000 cases and 5,000 controls) and a GRR of 5 for homozygote carriers. A reduction of $\mathrm{p}_{\mathrm{C}}$ to 0.001 from the reference scenario does not affect the relative decrease of $\log _{10} \mathrm{BF}$ and excess FRR, but the discriminative performance of pseudo $\mathrm{R}^{2}$ and $-\log _{10}$ Pval deteriorates. The excess FRR separates best for more common causal variants with $\mathrm{p}_{\mathrm{C}}$ equal to 0.05 and 0.2 . The $\log _{10} \mathrm{BF}$, pseudo $\mathrm{R}^{2},-\log _{10}$ Pval and excess FRR show similar reductions for $\mathrm{p}_{\mathrm{C}}=0.5$. Differing values of $\mathrm{D}^{\prime}$, sample size or prior precision show little impact on the advantage of the FRR. A reduction of $\mathrm{D}^{\prime}$ to 0.90 , which constrains values of $r^{2}$, results in a lower PAF for the marker than for the causative variant, and improves the separation by $\log _{10} \mathrm{BF}$, pseudo $\mathrm{R}^{2}$ and $-\log _{10} \mathrm{Pval}$ but does not affect the performance of the excess FRR. A smaller sample size (1,000 cases and 1,000 controls) decreases the discriminative ability of pseudo $\mathrm{R}^{2}$ and $-\log _{10}$ Pval. The distinction between marker and causal variant by $\log _{10} \mathrm{BF}$ was virtually identical when the prior precision considered in the Bayesian regression was increased 1,000-fold.

The lower portion of table 1 investigates the robustness of the six investigated statistics and their discriminative performances in the presence of genotyping errors. Assuming genotyping errors are independent of the actual genotype (fourth scenario in table 1), an error rate of $5 \%$ results in an overestimated PAF $(13.33$ vs. $7.37=+81 \%)$ that decreases slightly with decreasing $\mathrm{r}^{2}$. The five remaining statistics are underestimated under this kind of genotyping error. The relative reduction of the statistic value is highest for the excess FRR. A genotyping error of $5 \%$ for heterozygotes shows a smaller impact on the six statistics, but the discriminative ability is still greatest for the excess FRR.

\section{Illustration of Theoretical Results Based on Real \\ Datasets}

The values of the six statistics for the polymorphisms investigated by Edwards et al. [17] are shown in figure 4 . Only the excess FRR correctly identified SNP rs1061170 as the causal variant (the values of the six statistics for rs1061170 are represented by black dots). The functional SNP (rs1061170) ranked third according to AUC and pseudo $\mathrm{R}^{2}$, fourth according to $\log _{10} \mathrm{BF}$ and $-\log _{10} \mathrm{Pval}$ and eleventh according to the PAF.

The statistics for directly typed and imputed genotypes in the breast cancer study of Dünnebier et al. [21] are shown in figure 5. This example illustrates the unequal ranking of SNPs when different statistics are applied to a real dataset and imputed genotypes are investigated. The maximum AUC (0.61), pseudo $\mathrm{R}^{2}(0.034)$ and $-\log _{10} \mathrm{Pval}(1.88)$ were reached by SNP rs7187167. The maximum PAF (35), $\log _{10} \mathrm{BF}$ (2.72) and excess FRR (1.15) were attained by rs8052688, with $-\log _{10} \mathrm{Pval}=1.54$.

\section{Discussion}

GWA studies are contributing substantially to our knowledge of the genetic basis of human disease but the proportion of explained heritability for complex diseases remains modest, at best, for most traits [22]. For example, in a meta-analysis on type 2 diabetes, Zeggini et al. [23] identified 18 common variants that only explain about $6 \%$ of the increased risk of disease among relatives. The problem of 'missing heritability' is being hotly debated and it is likely that part of the heritability lies in large variants (deletions, duplications, inversions), epistatic and parent-of-origin effects, additional layers of genetic variation (microRNA, DNA methylation, histone modification) and rare variants [24, 25]. For example, Stefansson et al. [26] identified three rare deletions associated with schizophrenia, and susceptibility to autism, mental retardation and epilepsy has been shown to be modified by rare structural variants [27]. Some risk variants for cancer and type 2 diabetes only confer risk when inherited from a specific parent [28]. There are currently few examples of common variants that interact with each other or with the environment and account for heritability. We agree on the importance of considering multiple sources of genetic variation and of producing new data to investigate these hypotheses, but the importance of developing and applying novel statistical methods to the immense amount of existing data from data repositories should not be underestimated. 


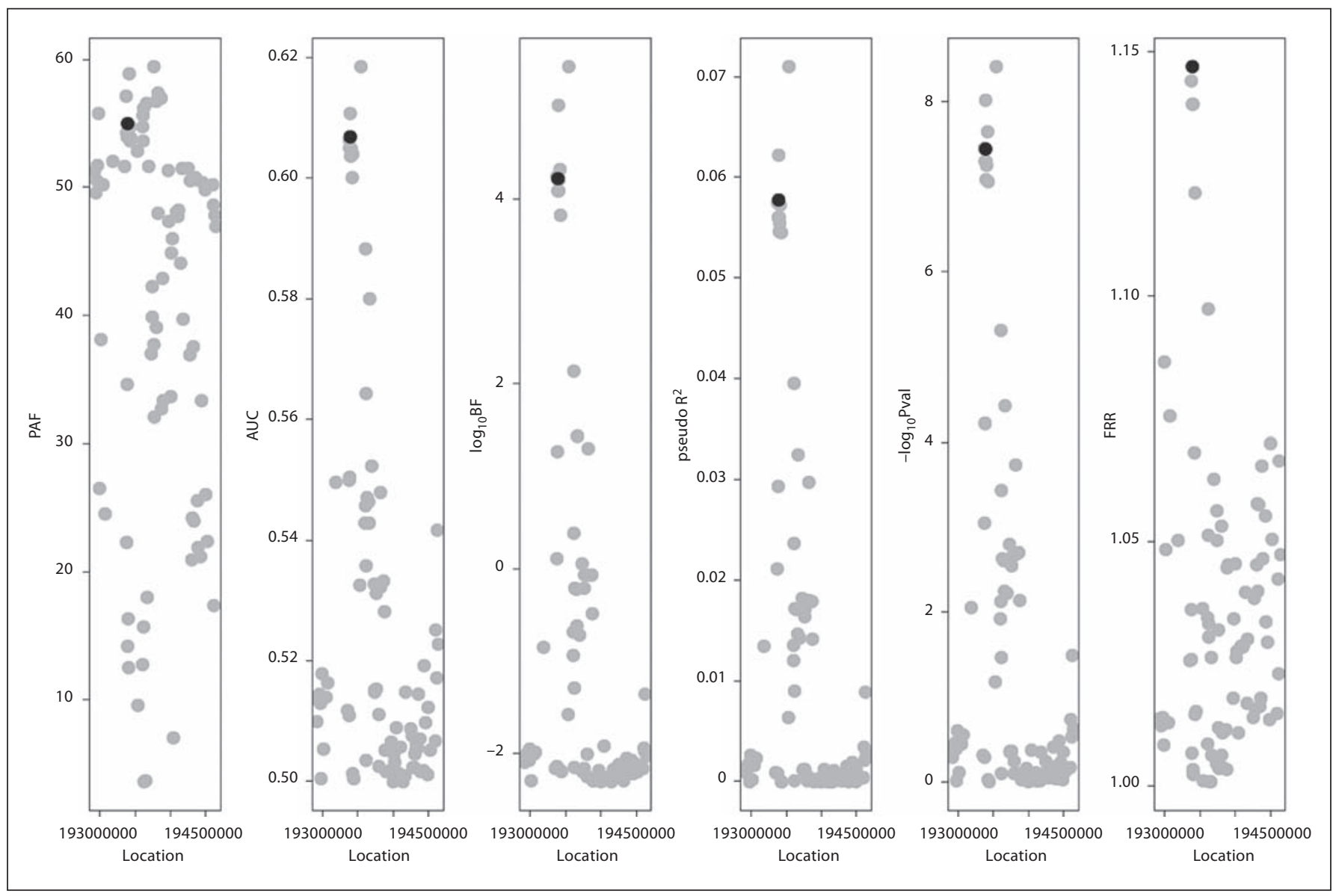

Fig. 4. Statistics of genetic association based on the supplementary material provided by Edwards et al. [17]. Values for SNP rs1061170 are represented by black dots.

This paper focuses on assessing alternative statistics that may facilitate the identification of causal or at least improved surrogate variants that will significantly contribute to heritability. So far, the underlying causal variants of polymorphisms with demonstrated disease associations have rarely been found and few disease markers associate with gene expression. We recommend inspecting FRRs to rank SNPs within association hotspots. For example, in the analysis summarized in figure 4, the SNP with the highest $-\log _{10} \mathrm{Pval}$ ( $\mathrm{rs} 1805287$ ) had a very low FRR ( 1.00). In other words, the variant with the highest $-\log _{10}$ Pval did not seem to contribute to the missing heritability of age-related macular degeneration. By contrast, the functional variant rs1061170 ranked only fourth according to $-\log _{10} \mathrm{Pval}$ but first according to the excess FRR.

Although PAFs are often reported in GWA studies to characterize the relevance of the identified variants, it should be kept in mind that PAFs are non-additive when risk factors are overlapping, as is clearly the case for genetically linked markers and causal variants. The PAF can be interpreted as the contribution of the identified variant to sporadic disease, whereas the FRR reflects its attributable proportion of familial cases. An interesting result from the present investigation is the similarity of the PAFs and, at the same time, the important differences between FRRs for markers and causal variants.

Pvals are the most common way of measuring association signals from GWA studies, but they have important disadvantages compared to BFs [29]. Pvals are often misinterpreted as the probability of no association given the observed data, but they actually yield the probability of the data given no association. The ranking of markers based on association Pvals is difficult to interpret, since Pvals depend on factors that influence power, such as allele frequency and sample size. In addition to these limi- 


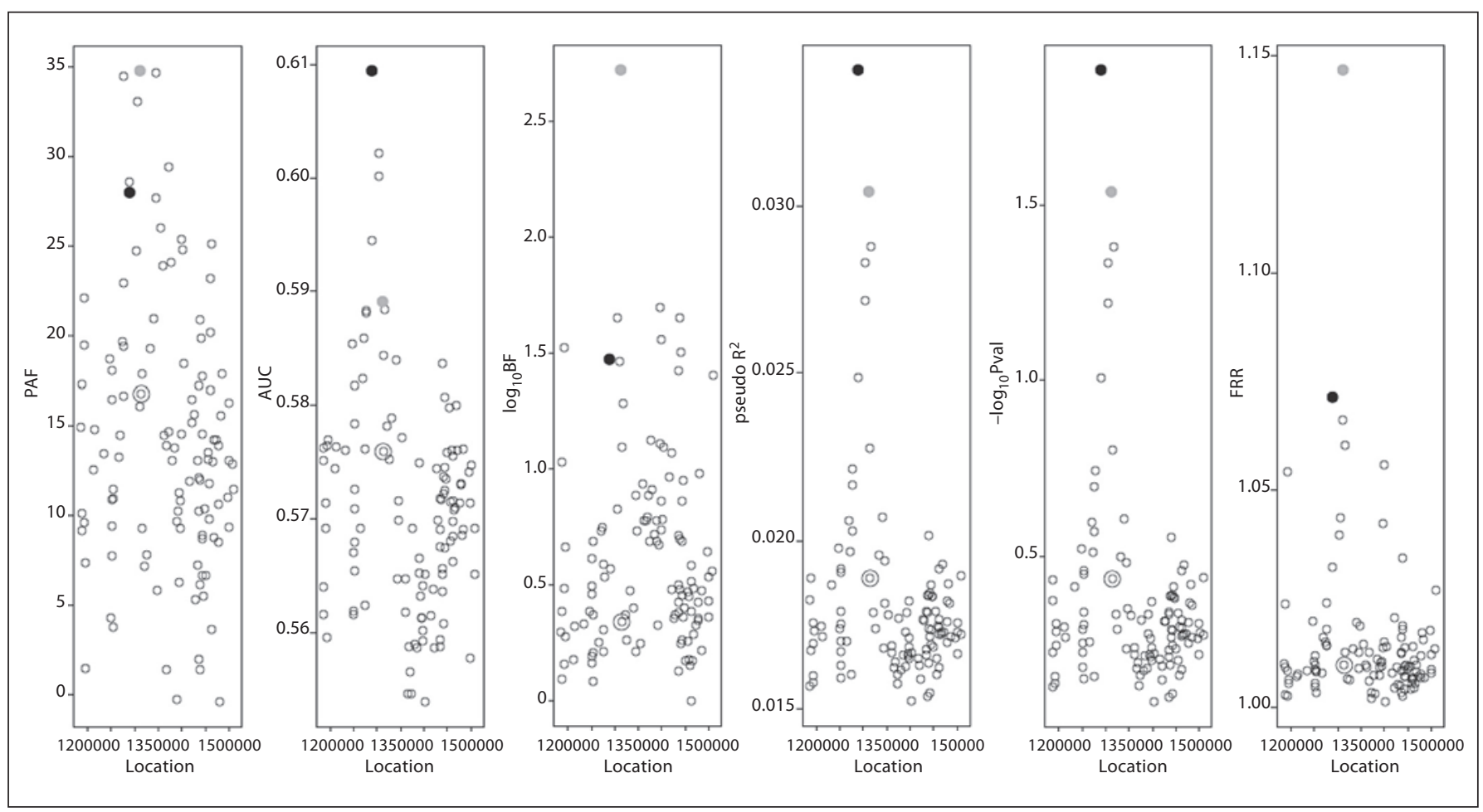

Fig. 5. Statistics of the genetic association for a region on chromosome 16. Small dots represent imputed genotypes, values for the three directly genotyped variants are represented by bigger dots (rs7187167 = black, rs8052688 = grey, rs11248866 = open). The maximum $-\log _{10}$ Pval $(1.88)$ was reached by SNP rs7187167. The maximum FRR (1.15) and the maximum $\log _{10} \mathrm{BF}(2.72)$ were attained by SNP rs8052688 $\left(-\log _{10}\right.$ Pval $\left.=1.54\right)$.

tations, we show here that BFs generally discriminate better between causal variants and markers than Pvals. The calculation of the two statistics for two real datasets resulted in similar rankings under moderate penetrance (per allele $\mathrm{OR}=2.7$, fig. 4 ) and different rankings with lower penetrances (fig. 5).

There is evidence that genetic association studies may benefit from exploiting existing catalogs of variation [15]. In order to extract the strongest signal of association, imputation methods are applied to model LD among variants in a panel of known haplotypes, such as the HapMap or the 1000 Genomes Project. Linkage information is then extrapolated to infer the genotype of untyped variants in individuals who are genotyped for a smaller number of variants. Some imputation methods consider phenotype information, others use multiple flanking SNPs and coalescence models to predict unobserved genotypes [30]. Consideration of phenotypes may improve the ability of imputation to separate causal from non-causal associations in the neighbourhood of a true risk variant, but comparisons have relied on Pvals thus far [31]. Imputation is not the main focus of the present article, but we use practical data to demonstrate that the ranking of variants, both imputed and directly typed, may depend on the statistics used. It would be very interesting to compare different imputation methods in combination with the six statistics explored here.

Resequencing efforts based on next generation technologies usually concentrate on chromosomal regions genetically close to the association signal. Dickson et al. [32] have shown that rare, high-penetrant causal variants located megabases away from the association signal can easily create genome-wide significant associations, a special case of indirect association they denominate 'synthetic association'. The authors recommend considering the possibility of synthetic associations in following up results from GWA studies and they observe stronger association statistics (Pvals) for the causal sites than for the marker. We have demonstrated in this paper that alternative statistics can also boost the identification of causal variants that may well lie within a much larger region than the LD block surrounding the associated, common variant.

Complete-genomic and even complete-exome sequencing is still expensive and sequencing familial cases 
has been proposed to enrich the frequency of rare variants. The FRR statistic indirectly takes this strategy into account: causal variants are inherited and they result in familial aggregation of disease. Another concern with next generation sequencing is genotyping errors, which substantially dilutes the evidence of association, particularly for rare variants. Although a comprehensive investigation of genotyping errors is beyond the scope of this article, we found the excess FRR to be more affected by relatively high error rates (e.g. 1.09/1.13 $=69 \%$ decrease) than other statistics (e.g. $24.3 / 55.7=44 \%$ for $\log _{10} B F$ ), but the discriminative ability of the FRR remained greatest under high genotyping error rates (13\% compared with $6 \%$ for $\log _{10} \mathrm{BF}$ ). A complete ordering of statistics according to discriminative performance for any scenario does not seem possible, but the excess FRR and the $\log _{10} \mathrm{BF}$ showed good properties. Advantages of the FRR over the $\log _{10} \mathrm{BF}$ are the interpretability of the statistic and the consideration of a fundamental assumption in genetic epidemiology: genetic variants are transmitted from parents to the offspring. If they really associate with disease susceptibility, they should result in the clustering of cases within families.

In conclusion, present data suggest that, under high penetrance, representation of genetic association by FRRs and BFs instead of Pvals may facilitate the distinction between causal and non-causal variants. The excess FRR showed the best discriminative power under most investigated scenarios, but no single statistic performed best in all situations. For example, rare moderate-penetrance variants (allele frequency $1 \%$, dominant $\mathrm{OR}=2.0$ ) seem to be best discriminated by BFs. The consideration of alternative statistics of genetic association may improve the reliability of association signals from studies that take advantage of next generation sequencing and/or multiple imputation based on public data repositories.

\section{Acknowledgment}

This work was supported by a grant from the Deutsche Forschungsgemeinschaft (DFG, SFB/TRR77, project Z2).

\section{Appendix}

Let $\mathrm{p}_{\mathrm{C}}$ represent the frequency of a causative allele $\mathrm{C}$ in $\mathrm{LD}$ with a more common marker allele $\mathrm{M}$. If $\mathrm{D}^{\prime}$ denotes Lewontin's measure of $\mathrm{LD}$ and $\mathrm{r}$ the correlation coefficient between $\mathrm{C}$ and $\mathrm{M}$, the frequency of $\mathrm{M}$ is

$$
\mathrm{p}_{\mathrm{M}}=\left[\mathrm{r}^{2}\left(1-\mathrm{p}_{\mathrm{C}}\right) /\left(\mathrm{p}_{\mathrm{C}} \mathrm{D}^{\prime 2}\right)+1\right]^{-1} \text {. }
$$

Discrimination between Causal Variants and Linked Markers by Six Statistics
The expected distribution of haplotypes is

$$
\begin{aligned}
& \operatorname{Pr}(\mathrm{CM})=\mathrm{p}_{\mathrm{M}} \mathrm{p}_{\mathrm{C}}+\mathrm{d}, \\
& \operatorname{Pr}(\mathrm{Cm})=\left(1-\mathrm{p}_{\mathrm{M}}\right) \mathrm{p}_{\mathrm{C}}-\mathrm{d}, \\
& \operatorname{Pr}(\mathrm{cM})=\mathrm{p}_{\mathrm{M}}\left(1-\mathrm{p}_{\mathrm{C}}\right)-\mathrm{d} \text { and } \\
& \operatorname{Pr}(\mathrm{cm})=\left(1-\mathrm{p}_{\mathrm{M}}\right)\left(1-\mathrm{p}_{\mathrm{C}}\right)+\mathrm{d},
\end{aligned}
$$

where $\mathrm{d}$ is the degree of $\mathrm{LD}$ (i.e. $\left.\mathrm{d}=\operatorname{Pr}(\mathrm{CM})-\mathrm{p}_{\mathrm{M}} \mathrm{P}_{\mathrm{C}}\right)$. The probability that an individual with genotype $\mathrm{cM} / \mathrm{cM}$ is affected is denoted by $\kappa_{0}=\operatorname{Pr}(\mathrm{A}=1 \mid \mathrm{G}=\mathrm{cM} / \mathrm{cM})$. Due to the assumption that conditional on causal genotype, the marker genotype has no effect on disease, we also have

$$
\begin{aligned}
& \operatorname{Pr}(\mathrm{A}=1 \mid \mathrm{G}=\mathrm{cM} / \mathrm{cM})=\operatorname{Pr}(\mathrm{A}=1 \mid \mathrm{G}=\mathrm{cM} / \mathrm{cm}) \\
& =\operatorname{Pr}(\mathrm{A}=1 \mid \mathrm{G}=\mathrm{cm} / \mathrm{cm})=\kappa_{0} .
\end{aligned}
$$

The relative risk of disease for homozygote carriers of $\mathrm{C}$ compared to wild-type genotypes is

$$
\mathrm{GRR}_{\mathrm{C} \_ \text {Hom }}=\operatorname{Pr}(\mathrm{A}=1 \mid \mathrm{G}=\mathrm{CC}) / \operatorname{Pr}(\mathrm{A}=1 \mid \mathrm{G}=\mathrm{cc})
$$

and the relative risk for heterozygotes compared to wild types is

$$
\mathrm{GRR}_{\mathrm{C} \_ \text {Het }}=\operatorname{Pr}(\mathrm{A}=1 \mid \mathrm{G}=\mathrm{Cc}) / \operatorname{Pr}(\mathrm{A}=1 \mid \mathrm{G}=\mathrm{cc}) .
$$

Therefore the probability that an individual has the genotype $\mathrm{CM} / \mathrm{CM}$ and is affected is given by

$$
\begin{aligned}
& \operatorname{Pr}(\mathrm{A}=1, \mathrm{G}=\mathrm{CM} / \mathrm{CM})=\kappa_{0} \mathrm{GRR}_{\mathrm{C}_{-} \text {Hom }} \operatorname{Pr}(\mathrm{G}=\mathrm{CM} / \mathrm{CM}) \\
& =\kappa_{0} \mathrm{GRR}_{\mathrm{C}_{-} \text {Hom }} \operatorname{Pr}(\mathrm{CM})^{2} .
\end{aligned}
$$

Similarly,

$$
\begin{aligned}
& \operatorname{Pr}(\mathrm{A}=1, \mathrm{G}=\mathrm{CM} / \mathrm{Cm})=\kappa_{0} \mathrm{GRR}_{\mathrm{C} \_ \text {Hom }} 2 \operatorname{Pr}(\mathrm{CM}) \operatorname{Pr}(\mathrm{Cm}), \\
& \operatorname{Pr}(\mathrm{A}=1, \mathrm{G}=\mathrm{Cm} / \mathrm{Cm})=\kappa_{0} \mathrm{GRR}_{\mathrm{C} \_ \text {Hom }} \operatorname{Pr}(\mathrm{Cm})^{2} \text {, } \\
& \operatorname{Pr}(\mathrm{A}=1, \mathrm{G}=\mathrm{CM} / \mathrm{cM})=\kappa_{0} \mathrm{GRR}_{\mathrm{C} \_ \text {Het }} 2 \operatorname{Pr}(\mathrm{CM}) \operatorname{Pr}(\mathrm{cM}), \\
& \operatorname{Pr}(\mathrm{A}=1, \mathrm{G}=\mathrm{CM} / \mathrm{cm})=\kappa_{0} \mathrm{GRR}_{\mathrm{C}_{-} \mathrm{Het}_{2}} 2 \operatorname{Pr}(\mathrm{CM}) \operatorname{Pr}(\mathrm{cm}) \text {, } \\
& \operatorname{Pr}(\mathrm{A}=1, \mathrm{G}=\mathrm{Cm} / \mathrm{cM})=\kappa_{0} \mathrm{GRR}_{\mathrm{C} \_ \text {Het }} 2 \operatorname{Pr}(\mathrm{Cm}) \operatorname{Pr}(\mathrm{cM}), \\
& \operatorname{Pr}(\mathrm{A}=1, \mathrm{G}=\mathrm{Cm} / \mathrm{cm})=\kappa_{0} \mathrm{GRR}_{\mathrm{C}} \mathrm{Het}_{2} \operatorname{Pr}(\mathrm{Cm}) \operatorname{Pr}(\mathrm{cm}), \\
& \operatorname{Pr}(\mathrm{A}=1, \mathrm{G}=\mathrm{cM} / \mathrm{cM})=\kappa_{0} \operatorname{Pr}(\mathrm{cM})^{2} \text {, } \\
& \operatorname{Pr}(\mathrm{A}=1, \mathrm{G}=\mathrm{cM} / \mathrm{cm})=\kappa_{0} 2 \operatorname{Pr}(\mathrm{cM}) \operatorname{Pr}(\mathrm{cm}) \text { and } \\
& \operatorname{Pr}(\mathrm{A}=1, \mathrm{G}=\mathrm{cm} / \mathrm{cm})=\kappa_{0} \operatorname{Pr}(\mathrm{cm})^{2} \text {. }
\end{aligned}
$$

The previous equations can be used to calculate the disease prevalence as a function of the marker genotype

$$
\begin{aligned}
\kappa_{\mathrm{MM}}= & {[\operatorname{Pr}(\mathrm{G}=\mathrm{CM} / \mathrm{CM}, \mathrm{A}=1)+\operatorname{Pr}(\mathrm{G}=\mathrm{CM} / \mathrm{cM}, \mathrm{A}=1)} \\
& +\operatorname{Pr}(\mathrm{G}=\mathrm{cM} / \mathrm{cM}, \mathrm{A}=1)] /\left[\operatorname{Pr}(\mathrm{CM})^{2}+2 \operatorname{Pr}(\mathrm{CM}) \operatorname{Pr}(\mathrm{cM})\right. \\
& \left.+\operatorname{Pr}(\mathrm{cM})^{2}\right], \\
\kappa_{\mathrm{Mm}}= & {[\operatorname{Pr}(\mathrm{G}=\mathrm{CM} / \mathrm{Cm}, \mathrm{A}=1)+\operatorname{Pr}(\mathrm{G}=\mathrm{CM} / \mathrm{cm}, \mathrm{A}=1)} \\
& +\operatorname{Pr}(\mathrm{G}=\mathrm{Cm} / \mathrm{cM}, \mathrm{A}=1)+\operatorname{Pr}(\mathrm{G}=\mathrm{cM} / \mathrm{cm}, \mathrm{A}=1)] / \\
& {[2 \operatorname{Pr}(\mathrm{CM}) \operatorname{Pr}(\mathrm{Cm})+2 \operatorname{Pr}(\mathrm{CM}) \operatorname{Pr}(\mathrm{cm})+2 \operatorname{Pr}(\mathrm{Cm})} \\
& \operatorname{Pr}(\mathrm{cM})+2 \operatorname{Pr}(\mathrm{cM}) \operatorname{Pr}(\mathrm{cm})] \text { and } \\
\mathrm{\kappa}_{\mathrm{mm}}= & {[\operatorname{Pr}(\mathrm{G}=\mathrm{Cm} / \mathrm{Cm}, \mathrm{A}=1)+\operatorname{Pr}(\mathrm{G}=\mathrm{Cm} / \mathrm{cm}, \mathrm{A}=1)} \\
& +\operatorname{Pr}(\mathrm{G}=\mathrm{cm} / \mathrm{cm}, \mathrm{A}=1)] /\left[\operatorname{Pr}(\mathrm{Cm})^{2}+2 \operatorname{Pr}(\mathrm{Cm}) \operatorname{Pr}(\mathrm{cm})\right. \\
& \left.+\operatorname{Pr}(\mathrm{cm})^{2}\right] .
\end{aligned}
$$

The genotype relative risks attributable to the marker are thus

$$
\begin{aligned}
& \mathrm{GRR}_{\mathrm{M}_{-} \text {Hom }}=\kappa_{\mathrm{MM}} / \kappa_{\mathrm{mm}} \text { and } \\
& \mathrm{GRR}_{\mathrm{M}_{\text {_Het }}}=\kappa_{\mathrm{Mm}} / \kappa_{\mathrm{mm}} .
\end{aligned}
$$




\section{References}

1 Manolio TA, Collins FS, Cox NJ, Goldstein DB, Hindorff LA, Hunter DJ, McCarthy MI, Ramos EM, Cardon LR, Chakravarti A, Cho JH, Guttmacher AE, Kong A, Kruglyak L, Mardis E, Rotimi CN, Slatkin M, Valle D, Whittemore AS, Boehnke M, Clark AG, Eichler EE, Gibson G, Haines JL, Mackay TF, McCarroll SA, Visscher PM: Finding the missing heritability of complex diseases. $\mathrm{Na}$ ture 2009;461:747-753.

$\checkmark 2$ Wakefield J: Bayes factors for genome-wide association studies: comparison with p values. Genet Epidemiol 2008;33:79-86.

3 Rosenthal R, Rosnow RL, Rubin DB: Contrasts and Effect Sizes in Behavioral Research: A Correlational Approach. Cambridge, New York, Cambridge University Press, 2000.

$\checkmark 4$ Killeen PR: Beyond statistical inference: a decision theory for science. Psychon Bull Rev 2006;13:549-562.

5 Strömberg U, Björk J, Vineis P, Broberg K, Zeggini E: Ranking of genome-wide association scan signals by different measures. Int J Epidemiol 2009;38:1364-1373.

-6 Risch N: Linkage strategies for genetically complex traits. I. Multilocus models. Am J Hum Genet 1990;46:222-228.

7 James JW: Frequency in relatives for an allor-none trait. Ann Hum Genet 1971;35:4749.

8 Lorenzo Bermejo J: Gene-environment interactions and familial relative risks. Hum Hered 2008;66:170-179.

9 Hemminki K, Lorenzo Bermejo J: Constraints for genetic association studies imposed by attributable fraction and familial risk. Carcinogenesis 2007;28:648-656.

-10 Hemminki K, Forsti A, Lorenzo Bermejo J: The 'common disease-common variant' hypothesis and familial risks. PLoS ONE 2008; 3:e2504.

-11 Nagelkerke NJD: A note on a general definition of the coefficient of determination. Biometrika 1991;78:691:692.

-12 Harrell FE Jr, Lee KL, Califf RM, Pryor DB, Rosati RA: Regression modelling strategies for improved prognostic prediction. Stat Med 1984;3:143-152.
13 Hosmer DW, Taber S, Lemeshow S: The importance of assessing the fit of logistic regression models: a case study. Am J Public Health 1991;81:1630-1635.

14 Hosmer DW, Hjort NL: Goodness-of-fit processes for logistic regression: simulation results. Stat Med 2002;21:2723-2738.

15 Marchini J, Howie B, Myers S, McVean G, Donnelly P: A new multipoint method for genome-wide association studies by imputation of genotypes. Nat Genet 2007;39:906-913.

16 Miclaus K, Chierici M, Lambert C, Zhang L, Vega S, Hong H, Yin S, Furlanello C, Wolfinger R, Goodsaid F: Variability in GWAS analysis: the impact of genotype calling algorithm inconsistencies. Pharmacogenomics J;10:324-335.

17 Edwards AO, Ritter R 3rd, Abel KJ, Manning A, Panhuysen C, Farrer LA: Complement factor $\mathrm{H}$ polymorphism and age-related macular degeneration. Science 2005;308:421-424.

18 Dünnebier T, Lorenzo Bermejo J, Haas S, Fischer HP, Pierl CB, Justenhoven C, Brauch H, Baisch C, Gilbert M, Harth V, Spickenheuer A, Rabstein S, Pesch B, Brüning T, Ko YD, Hamann U: Polymorphisms in the UBC9 and PIAS3 genes of the SUMO-conjugating system and breast cancer risk. Breast Cancer Res Treat 2010;121:185-194.

19 Dempster AP, Laird NM, Rubin DB: Maximum likelihood from incomplete data via the EM algorithm. J Roy Stat Soc Series B 1977;39:1-38.

20 Hivert MF, Manning AK, McAteer JB, Florez JC, Dupuis J, Fox CS, O’Donnell CJ, Cupples LA, Meigs JB: Common variants in the adiponectin gene (ADIPOQ) associated with plasma adiponectin levels, type 2 diabetes, and diabetes-related quantitative traits: the Framingham Offspring Study. Diabetes 2008;57:3353-3359.

21 Dünnebier T, Lorenzo Bermejo J, Haas S, Fischer HP, Pierl CB, Justenhoven C, Brauch H, Baisch C, Gilbert M, Harth V, Spickenheuer A, Rabstein S, Pesch B, Brüning T, Ko YD, Hamann U: Common variants in the UBC9 gene encoding the SUMO-conjugating enzyme are associated with breast tumor grade. Int J Cancer 2009;125:596-602.
22 Cirulli ET, Goldstein DB: Uncovering the roles of rare variants in common disease through whole-genome sequencing. Nat Rev Genet 2010;11:415-425

23 Zeggini E, Scott LJ, Saxena R, Voight BF, Marchini JL, Hu T, de Bakker PI, Abecasis $\mathrm{GR}$, et al: Meta-analysis of genome-wide association data and large-scale replication identifies additional susceptibility loci for type 2 diabetes. Nat Genet 2008;40:638-645.

-24 Eichler EE, Flint J, Gibson G, Kong A, Leal SM, Moore JH, Nadeau JH: Missing heritability and strategies for finding the underlying causes of complex disease. Nat Rev Genet 2010;11:446-450.

$>25$ Maher B: Personal genomes: the case of the missing heritability. Nature 2008;456:18-21.

26 Stefansson H, Rujescu D, Cichon S, Pietiläinen OP, Ingason A, Steinberg S, Fossdal R, Sigurdsson E, et al: Large recurrent microdeletions associated with schizophrenia. Nature 2008;455:232-236.

27 Stankiewicz P, Lupski JR: Structural variation in the human genome and its role in disease. Annu Rev Med 2010;61:437-455

28 Kong A, Steinthorsdottir V, Masson G, Thorleifsson G, Sulem P, Besenbacher S, Jonasdottir A, Sigurdsson A, et al: Parental origin of sequence variants associated with complex diseases. Nature 2009;462:868-874.

$>29$ Stephens M, Balding DJ: Bayesian statistical methods for genetic association studies. Nat Rev Genet 2009; 10:681-690.

30 Marchini J, Howie B: Comparing algorithms for genotype imputation. Am J Hum Genet 2008;83:535-539; author reply 539-540.

31 Lin DY, Hu Y, Huang BE: Simple and efficient analysis of disease association with missing genotype data. Am J Hum Genet 2008;82:444-452.

-32 Dickson SP, Wang K, Krantz I, Hakonarson $\mathrm{H}$, Goldstein DB: Rare variants create synthetic genome-wide associations. PLoS Biol 2010;8:e1000294. 